\title{
Cortex, countercurrent context, and dimensional integration of lifetime memory
}

\author{
Bjorn Merker \\ Department of Psychology, Uppsala University \\ Uppsala, Sweden
}

Draft version, slightly edited, of a paper published as:

Merker, B. (2004)

Cortex, countercurrent context, and dimensional integration of lifetime memory. Cortex, 40: 559-576.

The print version of this publication inadvertently omitted a few references noted at the end of the on-line version available through the Cortex website.

The following commentaries on the paper appeared in the same issue of the journal:

Finlay, B.L. (2004) The Calvinist cortex: Penetrating evolutionary predestination. Cortex, 40: 577-579, and

Lamme, V.A.F. (2004) Local versus global recurrence. Cortex, 40: 580-581, with a reply from the author: Merker, B. (2004) The local and the global in cortical phylogeny and function. Cortex, 40: 582-583.

Copyright (C) Björn Merker, 2004

Dr. Bjorn Merker

Gamla Kyrkv. 44

SE-14171 Segeltorp, Sweden

Phone: +46-86460860

E-mail: gyr694c@tninet.se 


\title{
Cortex, countercurrent context, and dimensional integration of lifetime memory.
}

Bjorn Merker

\begin{abstract}
:
The correlation between relative neocortex size and longevity in mammals encourages a search for a cortical function specifically related to the life-span. A candidate in the domain of permanent and cumulative memory storage is proposed and explored in relation to basic aspects of cortical organization. The pattern of cortico-cortical connectivity between functionally specialized areas and the laminar organization of that connectivity converges on a globally coherent representational space in which contextual embedding of information emerges as an obligatory feature of cortical function. This brings a powerful mode of inductive knowledge within reach of mammalian adaptations, a mode which combines item specificity with classificatory generality. Its neural implementation is proposed to depend on an obligatory interaction between the oppositely directed feedforward and feedback currents of cortical activity, in countercurrent fashion. Direct interaction of the two streams along their cortex-wide local interface supports a scheme of "contextual capture" for information storage responsible for the lifelong cumulative growth of a uniquely cortical form of memory termed "personal history." This approach to cortical function helps elucidate key features of cortical organization as well as cognitive aspects of mammalian life history strategies.
\end{abstract}

\section{Key words:}

Context, corticocortical connections, countercurrent, hippocampus, latent semantic analysis, life history strategies, neocortex. 


\section{Introduction}

Only mammals have expanded the dorsal pallial wall of the vertebrate forebrain into a multilayered and topographically organized sheet, the neo- or isocortex (Jerison, 1973; Kaas, 1995; Nieuwenhuys, 1994; see also Aboitiz, 1999; Marin-Padilla, 1998; and Striedter 1997). It is surrounded by and intimately interconnected with allocortical tissue. The cortical sheet as a whole is regionally differentiated into cortical areas (Brodmann, 1909; Felleman \& Van Essen, 1991) grouped into a small number of cortical domains (Young, 1993). The functional specialization of these areas suggests that cortex is organized in terms of task-specific modules (see, for example, Zeki, 1978; Livingstone and Hubel, 1988; Felleman and Van Essen, 1991). The same areas nevertheless exhibit a striking uniformity in structural arrangement at the cytoarchitectonic level, that is, normal to the cortical sheet (Creutzfeldt, 1977, 1978; Rockel, Hiorns and Powell, 1980; see also Somogyi, 1994, p. 149). This in turn suggests that cortical tissue performs a common function across compartments as diverse as sensory areas, motor cortex and zones of polymodal convergence (Braitenberg and Schüz, 1991). Few common functions are more easily compatible with such a wide range of specialization than one in the domain of information storage (Braitenberg, 1974).

In what follows, a specific conception of the functional organization of cortex will be used to suggest that a heterogeneous set of cortical attributes - attributes which include the phylogenetic correlation between relative isocortex size and longevity, patterns of inter-areal cortico-cortical connectivity, and laminar structure and interactions - are interpretable as inherent aspects of a unitary mechanism dedicated to the continuous accumulation and integration of permanent memory across the life-span. Under the name of "personal history" this form of uniquely cortical memory will be characterized, along with its implementation in a staggered countercurrent architecture ensuring deep contextual embedding of information for permanent storage (see Tulving, 1972; Craik and Tulving, 1975; Landauer \& Dumais, 1997).

\section{Isocortex size as a life history variable}

Mammalian brain size is an allometric correlate of body size (Jerison, 1973) as well as of longevity (Sacher, 1959). This makes it a member of a constellation of interrelated biological characters studied by evolutionary and behavioral ecologists under the name of life history variables (Charnov, 1993). Since both longevity (Sacher, 1976; Schmidt-Nielsen, 1975) and the size of organs within the body (SchmidtNielsen, 1975; Economos, 1980) scales with body size across species, a correlation between longevity and the size of an organ such as the brain or the adrenals need be no more than an allometric consequence of the scaling of body size with longevity (Economos, 1980).

Different parts or subdivisions of the brain do not, however, scale in the same manner with size. On a log-log plot relating the size of the brain's anatomical subdivisions to over-all brain size across species, isocortex exhibits both steeper slope and higher intercept than any other subdivision (Finlay and Darlington, 1995). That is, across mammalian species it makes a disproportionate contribution to brain size variance compared to other subdivisions of the brain. In so doing it does not lose the relation to 
longevity characterizing the brain as a whole. There is evidence in primates that relative isocortex size correlates with life-span across species. One study removed the effect of body size by the method of partial correlations, and found isocortex size to be the best predictor of longevity (Witkin, 1980), while another, relying on the method of linear regression residuals, found it to be second only to that other cortical structure, the cerebellum, in this regard (Hakeem et al., 1996; see also Allman, 1999, pp. 168175).

Even this neocortical relation to the life-span might be a passive reflection of body-size scaling, but such an interpretation provides no satisfactory explanation for why the neocortex specifically should be a privileged brain compartment in this regard. An alternative is to assume that relative neocortex size in fact determines body size through its impact on the species-specific life-span (cf. Hofman, 1993). A central determinant of species-specific life-span is the rate of external mortality impinging on a species in its ecological niche (see Charnov, 1993, and Section 4 and note ${ }^{1}$ ). If neocortical function acted to decrease rates of external mortality, and the magnitude of that effect depended on relative cortex size, then the large neocortices of some mammals would be a causal factor giving them the extended temporal horizons of external mortality under which they could grow slowly to large size. This construal is not tautological, since there are other ways of extending the temporal horizons of external mortality, such as by investing in the hard shell of the long-lived turtle or in the escape-capacity provided by flight in birds, who on average live more than twice as long as mammals of the same body weight (Lindstedt and Calder, 1976; see also discussion in Hakeem et al., 1996, p. 96).

There are a number of conceivable ways in which neocortex might affect rates of external mortality, but not all of these ways are equally compatible with a volumetric basis for that effect, as demanded by the correlations reviewed above. A promising candidate in this regard is the lifelong, continuous and cumulative storage of information in memory, since there are both theoretical and empirical grounds for believing that information storage is "space occupying", that is, that neural tissue is consumed ("used up") in the process of storing information. If so, an "information-storage" perspective on cortical function might account for the observed correlations on fundamental grounds.

\section{The volumetrics of information storage}

In order to last a lifetime memory must be deposited in irreversible forms of structural change, such as permanently changed patterns of synaptic efficacy. This means that there is a trade-off between plasticity and permanence/stability in the memory storage process (McCloskey and Cohen, 1989; Tucker and

1. The complex interrelationships among life history variables lie beyond the scope of this paper. A synthesis is available in Charnov (1993). In brief, species-specific life-span is an adaptation (Finch, 1990) to rates of external mortality (Medawar, 1952; Williams, 1957; Kirkwood and Holliday, 1986; Kirkwood and Rose, 1991) involving differential allocation of metabolic resources (Kleiber 1932, 1961; West et al., 1997, 1999; Banavar et al., 1999) to growth, maintenance and reproduction. These relations bear on the issue of memory in that temporal horizons of external mortality are not independent of an animal's knowledge of the circumstances in which it lives. 
Desmond, 1998). This trade-off becomes a "stability-plasticity dilemma" (Grossberg, 1987; Grossberg and Merrill, 1996, p. 259) only on the assumption that the same set of synapses must display both properties. The alternative is to irreversibly use up neural space as a medium for permanent memory storage by allocating new learning/memory to new tissue on a continuous basis. This solution resembles that of adding new units to an associative memory (Fahlman and Lebiere, 1990; Fahlman, 1991), with the difference that the "new units" are in place from the outset, to be used up sequentially over time. Such an arrangement would yield a volumetric correlate of lifetime storage capacity.

There are a number of indications that memory storage capacity is expressed volumetrically at the level of gross anatomy. The most direct evidence comes from studies that relate the size of specific subdivisions of the brain of birds to the learning and memory requirements of their behavioral adaptations. A number of species of birds hide food in multiple storage locations for later consumption. They therefore need to remember a multiplicity of spatial locations, and their hippocampus is relatively larger than in nonstoring species (Bingman et al., 1989; Harvey and Krebs, 1990; Krebs, 1990; Hampton et al., 1995; Healey et al., 1996; Volman et al., 1997; Patel et al., 1997). This volumetric effect is specifically associated with the persistence or permanence of memories (Biegler et al., 2001). The size of other forebrain structures in birds have also been related to learning capacity. For example, the relative size of the hyperstriatum ventrale predicts feeding innovation across 17 bird species (Lefebvre et al., 1997; Timmermans et al., 2000). Also, the relative size of the high vocal center (HVC, involved in song control) correlates with the number of song types typically found in the repertoire of 41 oscine species with learned song repertoires (Devoogd et al., 1993).

In mammals there is indirect evidence for a volumetric basis for information storage related to the need to remember for instrumental, ecological and social purposes. Passingham (1975) found that a measure of relative cortex size in primates correlates with performance on tasks of visual discrimination set as well as responsiveness to novel objects. Since novelty cannot be defined except by contrast with what is already familiar (Sachs, 1967; see also Sokolov, 1963) it involves the type of longitudinal and cumulative memory storage at issue here.

Ecological correlates of brain or neocortex size include diet in three orders of mammals: bats, rodents and primates (Eisenberg and Wilson, 1978; Pirlot and Stephan, 1970; Stephan et al., 1981; Clutton-Brock and Harvey, 1980; Mace et al., 1981; Harvey et al., 1980; Milton, 1988; Harvey and Krebs, 1990; Hakeem et al., 1996; Allman, 1999). When the effect of body size is removed, species feeding primarily on fruit generally are found to have larger brain or isocortex size than species feeding on insects (in the case of bats) or leaves (in the case of primates and rodents). The dietary effect has generally been interpreted in terms of the higher informational demands, including memory requirements, for locating fruit, which is high in energy content and easily digested but patchily distributed in space and time (see Allman, 1977, 1999; Eisenberg and Wilson, 1978; Harvey and Krebs, 1990; Hakeem et al., 1996). Social correlates of isocortex size have been reported for primates, and include group size (Dunbar, 1995; Barton, 1996; Joffe and Dunbar, 1997) and extent of intrasexual competition as indexed by sexual dimorphism and socionomic sex ratio (Sawaguchi, 1997). Presumably life in large groups and competitive interactions place a premium 
on long-term memory of the details of social interactions with many individuals. Interpreted in memory terms, these indirect lines of evidence regarding mammalian neocortex agree well with the direct evidence for the space-demanding nature of memory storage in birds (which lack a neocortex).

The indications that permanent neural storage of information is inherently space-occupying suggest a straightforward interpretation of the correlation between relative neocortex size and life-span in mammals. Were cortex an organ dedicated to the continuous and cumulative recording of memory, then the demand for neural storage space would bear a direct relation to the length of time over which the storage process extended. The correlation follows if that time is the species-specific life-span. The present treatment accordingly interprets cortex as an organ specialized for continuous (perpetual) and cumulative recording of permanent memory throughout the life span. The result is a unique, cortex-dependent, informational resource which brings a powerful mode of inductive knowledge within reach of neural implementation, a form of knowledge which holds the key to much of the secret of what is distinctly mammalian.

\section{The powers of perpetual storage}

From the point of view of the life-history argument pursued so far, the significance of lifelong cumulative memory recording bears on the issue of reducing the odds of external mortality in two principal ways, one pertaining to specific contents of memory, the other to their global statistics (the illustrative examples that follow are tailored to large-brained mammals, while cognitively less demanding analogs would apply to small-brained ones):

1. Only permanent memory allows its possessor to retain information concerning things like who did what to whom months, years or decades ago (Humphrey, 1976; de Waal, 1982; Byrne and Whiten, 1988). Above all, it is the only means for capitalizing on experience of statistically rare but exceptional or anomalous events and contingencies, situations which may not recur for years but memory for which may be highly advantageous. The utility of such memory increases with the sophistication with which its specific contents are defined and grasped. It provides a selection pressure, in other words, for expansion of the brain's representational capacity.

2. Many global ("higher order", "deeper") statistical invariants of environmental contingencies, such as long-term patterns of fruiting succession across diverse species and locations of trees, can be extracted from experience only over the long run of many seasons/cycles/years. That is, the very acquisition of knowledge of such statistical features of the environment requires permanent and cumulative memory storage over the same time horizons. In the limit, the trapping of information available only over a lifetime of exposure, such as patterns and consequences of generational succession or the adjustment of migratory routes to climatic or vegetation cycles (patterns which may span decades: Binford, 1983, pp. 114-117), requires lifetime permanence of cumulative memory storage for its implementation. The storage of information for the full length of the species-specific life-span is a prerequisite for the ultimate and fullest use of the postulated cortical capacity, though the resource of lifetime-long memory has many uses short of this ultimate use. The latter may, however, provide a selection pressure for extension of the life-span in 
mammals. There is potential for synergism between this selection pressure and that mentioned in point one above.

In terms of information, the two types of memory content just sketched appear to have very little in common except their dependence on long-term memory recording. One is concerned with historically specific and sometimes unique individual items and events, while the other concerns global statistical regularities extracted over many episodes, items or events. In fact, these two content modes exemplify a fundamental tradeoff between recording a variety of specific aspects (any one of which might be important) and redundant (statistically regular and hence important) aspects of noisy information (Haft, 1998). The potential advantage of integrating them was hinted at above, in noting that the utility of the item-specific type depends on the cognitive sophistication with which the recorded item or episode is grasped and interpreted. It makes a vast difference in this regard whether the memory of, say, a social encounter is stored as a record of bare sequences of body movements, or whether inferred motives of participants are included in its representation. Knowledge of motives presumably derives from ample experience with many such instances and their outcomes.

The key concept of the present paper is that global statistical knowledge of the kind featured in point 2 above provides the interpretive framework for the first, specific, type and that the two forms of memory contents in fact are only the two functional poles of a single unitary form of uniquely cortical inductive knowledge which combines item specificity with classificatory generality. To do so, such a form of memory would have to achieve global coherence in the sense that each of its content elements should be related to every other via its place and significance in a given subject's stored experiential history. This is most readily accomplished by organizing memory contents contextually, that is, by making storage of an item dependent on the context to which it belongs. Since broader, more general contexts include narrower, more specific ones, this amounts to a system of "nested contextual subsumption" with concept-like properties. The historical (temporal) aspect of such a scheme would in general terms follow from the already mentioned strategy of allocating new learning/memory to new tissue on a continuous basis, amounting to inclusion of time of acquisition (broadly conceived) as a contextual parameter of storage.

The unitary nature of the postulated mode of storage has analogs in schemes for extracting latent higher-order statistical structure from a first-order data-stream, such as "latent semantic analysis" (LSA, see Landauer \& Dumais, 1997; Landauer et al. 1998) and related approaches (Burgess et al., 1998; Lund \& Burgess, 1996; Burgess \& Lund, 2000; see also Hopfield, 1982; and Steedman, 1999). Though these approaches were developed for text analysis, Landauer \& Dumais (1997) have emphasized that the statistical principles they embody are likely to have domain general applications. These principles exploit the powers of multi-variate dimension-matching by mapping large amounts of transformed data onto a single unitary space of optimal dimensionality. In so doing they achieve a felicitous contextual relation between local and global information amounting to a form of semanticity. In a number of cases this has led to simulation results suggestive of human-like performance (Landauer \& Dumais, 1997).

The "complete connectivity" (in the neural network sense) and unitary nature of the multidimensional representational space featured in these programs ensures that the relation between any two content 
items is a function of the entirety of the contents of the representational space. When such a system works in time by accumulating its contents through sequential information exposure, the accretion of each new item potentially and marginally alters the representation of all other items (Landauer \& Dumais, 1997). Thus, the representation of any given item becomes a function of that of all other items, and every accretion adds to the semantic powers of the system. In fact, to achieve working capacity, the system needs to accumulate a considerable mass of naturally inter-related data (Landauer \& Dumais, 1997).

The example illustrates the potential powers of an architecture of memory that unifies the two contentdomains of personal history (roughly, its "episodic" and "semantic" poles) into a coherent framework for the inductive acquisition of knowledge based on perpetual memory recording. As will be developed in the rest of this paper, key features of cortical organization jointly seem to implement such a system of memory storage, bringing formidable powers of inductive knowledge to bear on mammalian adaptations.

\section{Cortical substrates for the accretion of personal history}

So far the neocortex has been considered as a unit, in simple volumetric terms. However, as already mentioned, the cortical sheet is composed of anatomically distinguishable subregions exhibiting functional specialization featured in modular (Zeki, 1978; Livingstone and Hubel, 1988) and hierarchical (Felleman and Van Essen, 1991) conceptions of cortical organization. Since cortical cells do not exhibit striking areaspecific differences in membrane biophysics (Douglas \& Martin, 1991), and local cortical structure is rather uniform across areas (see references in introduction) the causal basis for this functional specialization must be sought largely in the differential external afference of cortical areas on the one hand, and in the pattern of inter-areal fiber connections on the other (on this latter point, see Young, 1993, p. 18 and Sections $5 a, b$ and c, below). The former pertains of course above all to the primary sensory areas of cortex. In addition to being distinguished by their receipt of the details of on-line sensory activity in a given modality, they exhibit distinct cytoarchitectonic characteristics (koniocortical heterotypic architecture) and a more restrictive deployment of cortico-cortical connections (Felleman and Van Essen, 1991; Young, 1993) than is typical of the far more numerous cortical areas which together comprise so called homotypic cortex. The primary areas, together with "tandem areas" such as area V2 in the primate visual system, are therefore best considered specialized "sensory staging areas" which supply cortex with high-resolution sensory input without themselves being typical members of the system of functional areas they supply. In what follows, generalizations about the cortical system will, unless otherwise noted, refer to characteristics of non-primary, homotypic cortex.

\section{5a. Inter-areal connectivity as a framework for contextual embedding}

Cortical areas are interconnected via a massive system of inter-areal cortico-cortical fibers travelling in the cortical white matter. The latter consists to greater than 95 percent of intracortical axons (Abeles, 1991), leaving less than 5 percent for the entirety of cortical input and output. This highly skewed ratio between extrinsic and intrinsic cortical connectivity means that cortex is a massively self-connected structure, as 
emphasized by Braitenberg (1977, p. 106) and as is macroscopically visible in the mushroomlike excrescence of the telencephalon on its narrow diencephalic stalk.

Cortical self-connectivity conforms to a "small-world" pattern of inter-connections among the set of anatomically and physiologically identified cortical areas (Stephan et al., 2000), each of which, without exception, is related to multiple other areas by direct and generally reciprocal and topographically organized axonal projections. In the well-studied case of the visual system of the macaque, each area is connected to between 10 and 15 other cortical areas (Felleman and Van Essen, 1991). Known connections between the 32 visual areas included in the Felleman and Van Essen analysis amount to 31 percent of all theoretically possible ones, a lower bound estimate which agrees well with the 37 and 35 percent estimates of the same parameter for the auditory and somatosensory-motor systems (Young, 1993). The agreement of these figures across domains is suggestive of lawfulness, possibly relating to the capacity peak at 30-40 percent connectivity found by simulation for a "convergence zone" model of memory (Moll and Miikulainen, 1997, Figure 5).

Small-world connectivity means that but a few cortico-cortical projection steps are required to link any given cortical area with any other (Stephan et al., 2000, pp. 114-115; Sporns et al., 2000), and thus to the full set of cortical areas. While not being "completely connected" in the direct sense of neural network models, cortex achieves a "staggered" global connectivity in but a few steps of cortico-cortical projection (to which is added the countercurrent contribution to global connectvity considered in Section $5 \mathrm{c}$ ). In this small-world connective context an area has a greater probability of being anatomically connected with close than with distant neighbors in the cortical sheet (see, e.g., Felleman \& Van Essen, 1991, fig. 5). This means that the connective relations of a given cortical area are related to the pattern of functional specialization among areas, because close areal neighbors in the cortical sheet are also likely to be functionally more similar than distant neighbors, a circumstance emphasized by graph theoretic analysis of connectional relations (Young, 1992, 1993, p. 18; Young et al., 1995; Burns and Young, 2000). Since cortico-cortical input to a given area exerts a powerful influence over local activity even when arriving over other than feed-forward projections to the granule layer (Mignard and Malpelli, 1991;Tomita et al., 1999; Lamme \& Spekreijse, 2000; see also Salin and Bullier, 1995 and Payne and Lomber, 1999), this means that every cortical area and every local point within it stands under the functional influence of activity converging on it from a sizeable set of other cortical areas on a gradient of diminishing functional similarity. That is, basic and quite general features of cortico-cortical connectivity subject functional activity at every point in the cortex to a form of direct, obligatory and graded contextual embedding.

The above two points can be summarized as follows: by virtue of elementary cortico-cortical connectivity alone, cortex exhibits staggered global connectivity with obligatory contextual organization. Two additional aspects of inter-areal connectivity will extend this conception by further defining the scope and nature of potential context effects at the cortical level.

\section{5b. Frontolimbic connectivity and contextual subsumption}

While the macaque visual, auditory and somatosensory-motor systems exhibit an inter-areal connective 
density amounting to around one third of all theoretically possible connections within each domain, the corresponding number for the cortical sheet as a whole is roughly half that, or 15 percent (Young, 1993). It follows that there must be cortical domains across whose borders inter-areal connectivity is sparser than within those borders. Such a "clustering" of cortical connectivity in fact defines the three sensory domains just referred to (Stephan et al., 2000; see also Tononi et al., 1994). All three, however, are heavily interconnected with a fourth domain defined on the basis of graph-theoretic analysis, and designated the frontolimbic domain (Young, 1993). Since each of the three "sensory" domains are more richly interconnected with the frontolimbic domain than with each other, the frontolimbic domain supplies the common connective center of gravity of the entire cortical system, as illustrated in Figure 1. The arrangement is epitomized in sites of global convergence such as the hippocampal formation and the anterior superior temporal polysensory area (Jones and Powell, 1970; Bruce et al., 1981; Damasio, 1989; Oram and Perret, 1994a).

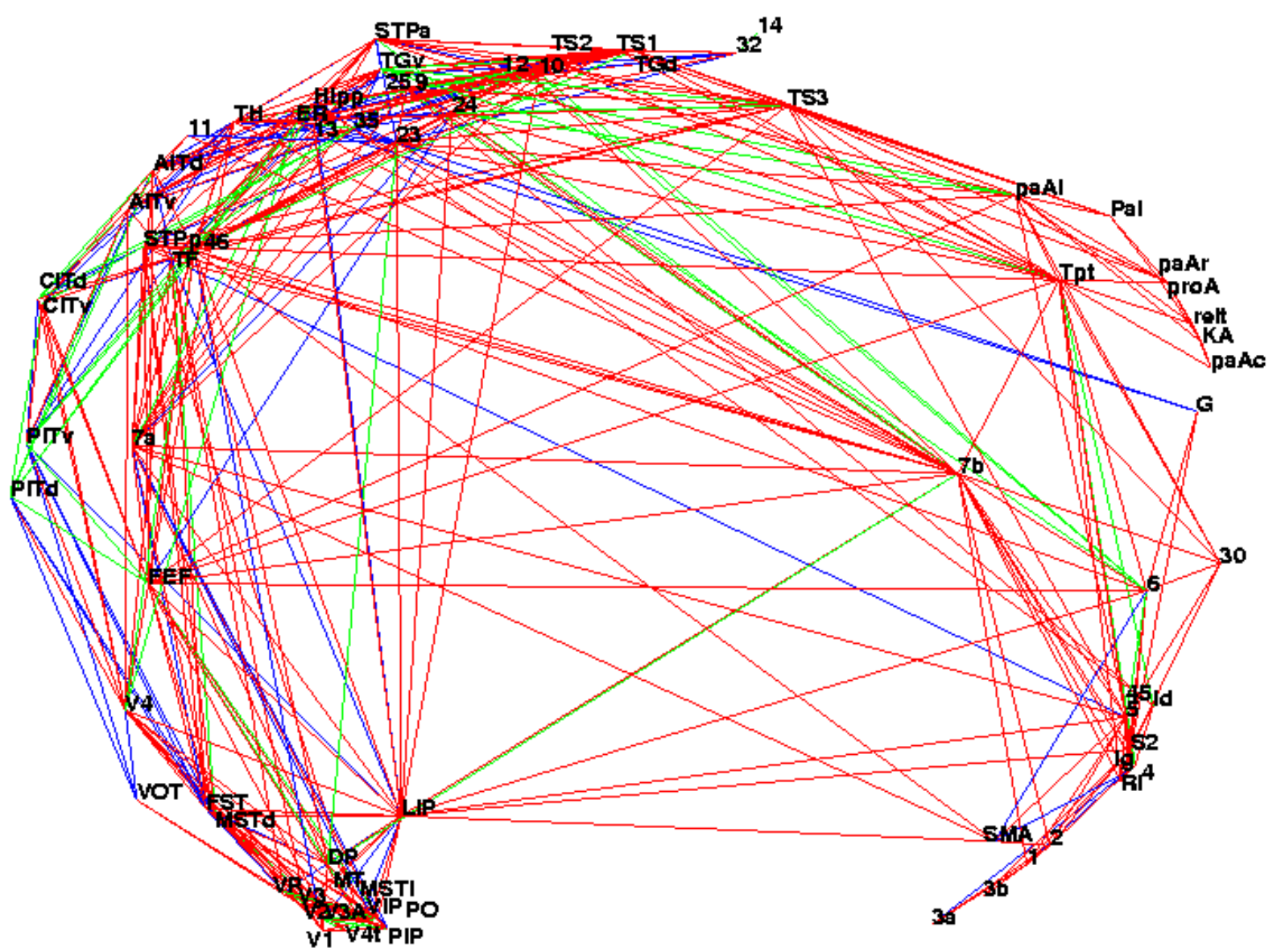

Fig. 1 - Connective relations of the cortical map system of the macaque, from Young (1995). Cortical areas are represented as labelled points. The degree of anatomical connectedness between areas is represented by their proximity in the plane of the figure. Sensory domains occupy the lower part of the figure, while the frontolimbic domain is at the top. The visual system is on the left, the somatosensory-motor on the lower right, the auditory on the upper right. In the visual domain, connections of "dorsal stream" areas with auditory and somatosensory areas split the visual system into two subdomains. Note that in connective terms the frontal eye fields are grouped with the visual areas of posterior cortex despite their frontal anatomical placement. Used with the permission of M. Young and The Royal Society. 
The ubiquity of reciprocal interareal connectivity and the dense interconnections of each of the three sensory domains with the densely self-connected frontolimbic domain provides paths linking any area or point in cortex with any other via the frontolimbic domain. The globalizing consequences of this arrangement are most readily evident when considering the connective arrangement of cortical areas independently of their placement in the cortical mantle. Individual cortical areas are two-dimensional topographic sheets and inter-areal projections tend to connect corresponding points in these topographies. Figure 2 (b and c) illustrates the simple operation of eliminating (minimizing) the fiber length
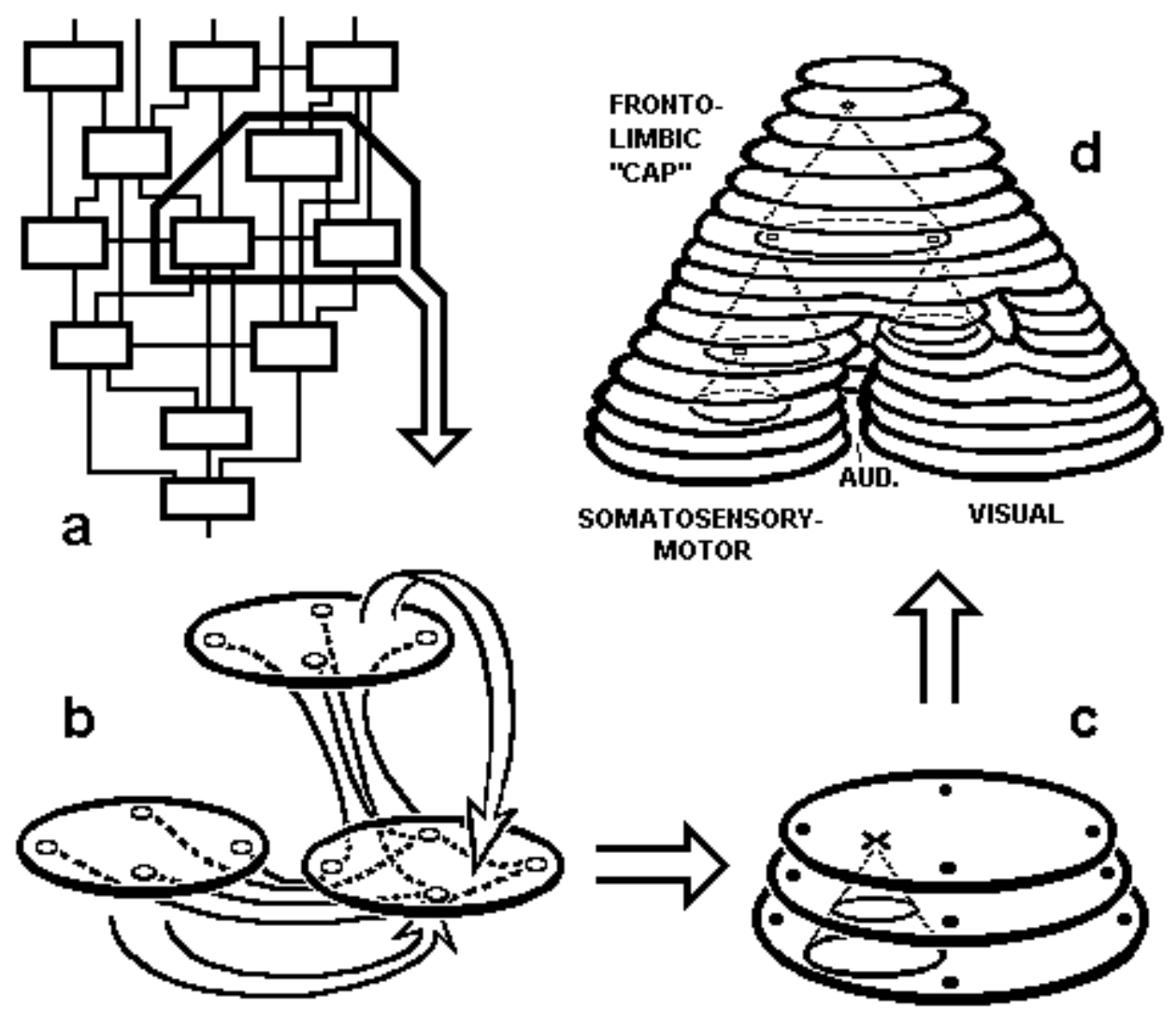

Fig. 2 - Highly schematic illustration of "connective consolidation" of the cortical map system into a unitary neural solid with pyramid architecture. a: Simple "cortical hierarchy" in which areas are represented by boxes and their connections by lines. b: Connections between three sample areas expanded to indicate the topography of their connections. c: Topographic superposition of areas in b through minimization of the length of all fiber connections between them. Convergence-divergence principle of functional connectivity indicated by cone. d: The operation shown in b and c generalized to the entire cortical map system generates a "neural solid" with global pyramid architecture.

of white matter connections linking any two connected cortical areas. Through juxtaposition of corresponding points in their areal topographies, the areas will superpose in topographic register. They do so whether they occupy the same or different levels of the cortical "hierarchy" (Figure 2b). The net effect is a superposition of areas in a "map stack" whose axis runs orthogonal to the plane of superposition. 
Systematically extended to all connected areas this concept generates a single "neural solid" 2 crowned by frontolimbic cortex and branching downwards into sensory domains (or modality "substreams"), as illustrated in Figure 2d.

Local convergence-divergence of connectivity (see Braitenberg, 1981; Mcllwain, 1986; Salin and Bullier, 1995) ensures that points on maps higher in the sequence tend to subsume and to have broader functional scope than their congeners earlier in the sequence. Within the sensory domains this is reflected in a broadening of receptive fields as one ascends the system (Van Essen, 1985; Felleman and Van Essen, 1991), as well as in a drastic diminution of areal size at high hierarchical levels (see Felleman and Van Essen, 1991). "Pyramid architectures" provide a conceptualization of these circumstances (see, e.g., Adelson et al., 1991, and references therein). The pattern of "subsumption" is reinforced by the broader local distribution of feedback projections (see Section $5 \mathrm{c}$, below) compared to feedforward ones (Salin and Bullier, 1995). The net effect of these structural features is that points high within a domain connectively and functionally subsume points located in a progressively expanding domain ("cone") beneath themselves (Figure $2 \mathrm{c}$ and d). Since inter-areal connections are largely reciprocal, there is a connective path between any two points within such a cone via a sufficiently high nodal point within the cone. The same holds for the apices of any pair of cones within the neural solid as a whole (see fig. 2d), and thus the "place systems" of the two-dimensional parameter spaces of individual cortical areas (Schwartz, 1984) are connectively integrated into a global representational space. No two points have the same functional content and all points are coherently related by nested contextual subsumption.

The neural solid as a whole thus emerges as an ordered contextual plenum, or unitary and coherent "epistemic space", for which connective path-length provides an implicit metric. The analogy already drawn to "latent semantic analysis" in section 4 above raises the question of the dimensionality of this neural space. That problem awaits formal solution, but should be tractable, along the following heuristic lines. Assuming that human language captures a good part of the useful structure of the world, the 200 to 300 dimensions that have proven optimal for latent semantic analysis are probably not far removed from the number of dimensions required by the brain's epistemic space itself, on which our liguistic capacities necessarily depend in a most intimate fashion. Each cortical area is functionally specialized, and thus supplies an informational dimension. Its information content, however, takes the form of a two-dimensional parameter space (Schwartz, 1984). Two times the number of cortical areas would accordingly be a starting point for refining dimensional estimates by correcting for less than total connectivity - which reduces dimensionality - and for hemispheric specialization - which increases dimensionality. For the macaque with some 70 areas that gives a starting benchmark of 140 dimensions, and for humans with some 100 areas or more the number would be at least 200 . This brings the 200 to 300 dimensions found

2. The exact vertical position of any given cortical area in relation to its neighbors in the neural solid is underconstrained by laminar connective criteria of the type used by Felleman and Van Essen (1991) to arrange visual areas in a hierarchy. The combinatorial consequences of local alternative placements of many areas (independently of one another) leads to the large total number of alternative hierarchical arrangements obtained in the analysis of Hilgetag et al. (1996). These details do not affect the over-all scheme of the "neural solid", whose layering sequence is locally indeterminate within a global ordering. 
to be optimal in simulation runs of latent semantic analysis at least within reach. Additional factors bearing on estimates of a cortical dimensionality are the extent to which non-primary cortical areas share sensory topographies, columnar representation of multiple functions within an area, and finally the laminar organization of corticocortical projections into two oppositely directed "streams". They span the entire cortical expanse from sensory areas to frontolimbic cortex in both directions, and reinforce the global functional connectivity of the cortex through an arrangement amounting to a "countercurrent" system.

\section{5c. Countercurrent partitioning of cortical context}

As first described by Rockland and Pandya (1979) and elaborated by Felleman \& Van Essen (1991), distinctive patterns of laminar origin and termination of inter-areal connections define a directional "axis of transmission" across the set of cortical areas. To a first approximation, projections terminating in the middle, granular layers of cortex define a "feed-forward" direction, while those terminating in the flanking superficial and deep layers, define a "feedback" direction. Projections that are nonselective for layers define a "lateral" direction, orthogonal to the bidirectional axis defined by the other two patterns (Felleman and Van Essen, 1991). Feedforward projections connect a given area of cortex not only to its nearest neighbor in the forward direction, but to next-nearest and further areas, and not infrequently to frontolimbic cortex itself. Since the same is true of each of the intermediate areas to which it projects the result is a multi-limbed and staggered projective cascade in the feedforward direction illustrated in Figure $3 \mathrm{~b}$. The same arrangement holds for the feedback connections. A bidirectional multiply staggered projection cascade accordingly connects frontolimbic cortex and the primary sensory areas. The functional efficacy of the front-to-back current of cortical information flow (Teuber, 1978, p. 900) is reflected in phenomena
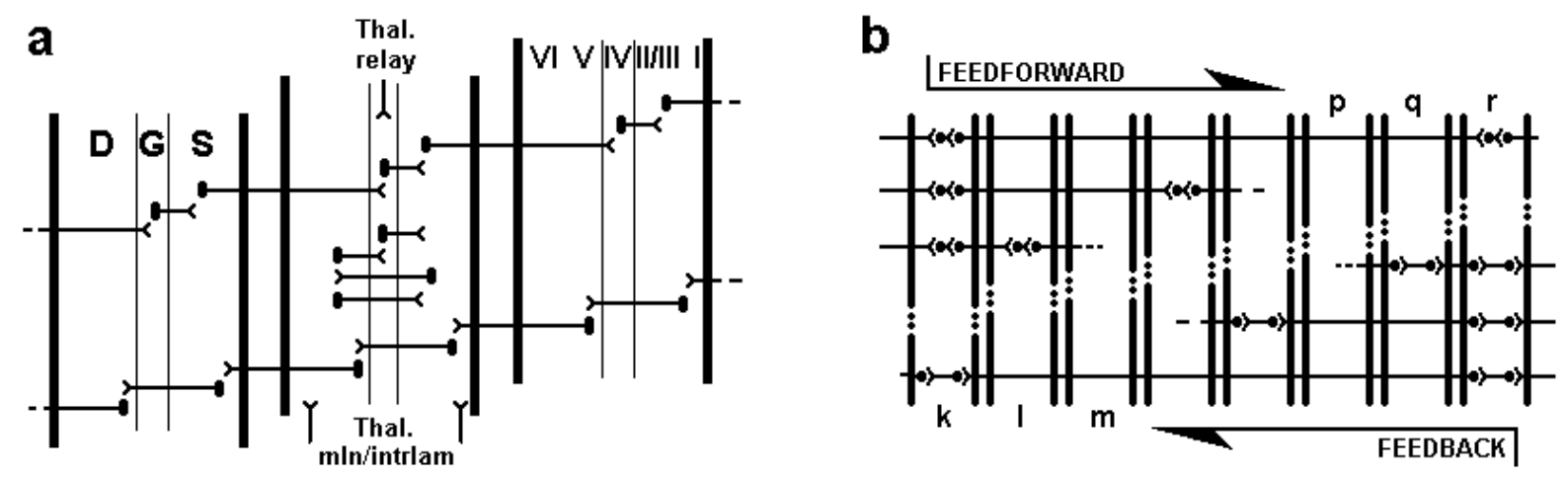

Fig. 3 - Connectional primitives of the cortical countercurrent system shown as they would appear after topographic superposition of patches of cortical areas in accordance with the conventions in Figure 2. a: Inter-areal connectional basis of the feedforward and feedback currents, highly schematized. D, G and S stand for deep, granular, and superficial cortical layers, respectively. A patch of cortex (middle) is shown in its laminar connective relationship with similar patches in flanking cortical areas directly connected to it. A few local interlaminar pathways are also schematically included, as are specific and "nonspecific" thalamic afferents. b: A given cortical area, such as area "k" or " $r$ " at the extremes of the figure, project in a staggered, overlapping fashion not only to their immediate neighbors but directly to more distant areas. Each of the intervening areas duplicates this multiple staggered connectivity, though this is not shown in the figure. The resulting multiply nested connectivity amounts to a projective cascade for relay-like sequential transfer of activity across areas. The bidirectional nature of this arrangement forms a countercurrent system due to laminar segregation of the two directional streams. 
such as the use of primary visual cortex by working memory for the temporary retention of visual detail (Supèr et al., 2001). Even low-level perceptual learning appears to recruit countercurrent involvement with increasing task difficulty (Ahissar and Hochstein, 1997).

The contextual connectivity of cortex is, in other words, partitioned into two laminarly segregated and oppositely directed complements or "currents" whose laminar specificity and segregation maintains their identity across cortical areas. They interact locally - and only locally ${ }^{3}$ - at every point of the cortical map system through a variety of local translaminar connections, epitomized in (but far from limited to, see Figure $3 a$ and Callaway, 1998) the translaminar trajectory of the apical dendrite of cortical pyramidal cells. These features of cortical organization conform to and instantiate a general class of biological mechanisms exhibiting bidirectional gradient design, namely countercurrent systems (see SchmidtNielsen, 1997; Deacon, 1989; Ullman, 1995; Schmidhuber, 1996). Their principal functional distinction is that of achieving global transformations by strictly local means (for which see Schmidhuber, 1996).

Countercurrent connectivity ensures that every patch of cortical tissue forms a permanent interface between two different streams of neural influence ultimately originating at opposite ends of the cortical map system. One issues from the primary sensory areas conveying high-resolution detail of current states of the world, while the other issues from frontolimbic domains of multimodal convergence representing high-level combinations of goals, motivations and experience. This functional polarity applies to each intermediate and less global step of the cascade as well, and may rest on fundamental informationtheoretic requirements (see Dayan et al., 1995; Friston 2002; Kawato et al., 1993; Rao and Ballard, 1997). The position of frontolimbic cortex at the head of this system ensures that this bidirectional connectivity by itself would suffice to globalize the connective structure of the cortex - placing the content of every patch of cortex under the ultimate influence of all the rest - though in the event it only further reinforces the contextual organization of cortex described in earlier sections.

The countercurrent principle is bound to play a central role in the accretion of personal history in several functionally linked ways: first, personal history is the stored residue of experience, which is populated by objects, events, and other contents of consciousness. That is, conscious awareness is the functional "gate" through which information enters personal history, and the neural conditions for conscious awareness at the cortical level appear to require interaction between the two currents (Lamme and Roelfsema, 2000; Lamme and Spekreijse, 2000). The essential idea of the intersection of the two activity streams in this regard is illustrated in the left panel of Figure 4 (next page). Moreover, the conversion of experience to stored personal history involves expectancy, anticipation, and their violations in fundamental ways (see "contextual capture" and "escape from containment" in Section $5 \mathrm{~d}$ and $5 \mathrm{e}$, below). For this, the

3. The laminar nonspecificity of "lateral" connections violates the strictly local interaction of the two streams, since laminar mixing takes place inter-areally via lateral connections. Since lateral connections run orthogonal to the principal directional axis defined by the two laminarly specific projection patterns, "laminar mixing" via lateral connections remains largely confined to "same-level neighbors" within a cortical domain (or subdomain in the visual system). Its effect is therefore to de-emphasize differences between lateral neighbors in the system, and this in turn underscores the unitary "stack" structure of a domain (or sub-domain) depicted in Figure 2d. 
feedback stream descending from frontolimbic cortex ("expectancy", "containment") and interacting locally along the way (in multiple locations) with the feedforward stream ascending from sensory areas (potential violations of expectancy, "escape from containment") would seem to provide an ideal mechanism (see also Dayan et al., 1995, and Friston 2002, for formal treatments of bidirectional processes in terms of the general concept of inference).

By analogy with other examples of countercurrent organization (vascular, renal), which conserve or even amplify a resource (heat in the vascular case and ionic gradients in the kidney), the cortical countercurrent may also act at a more elementary level to sustain neural activation levels as a means to effect lasting excitability changes in cortical synapses required for long-term storage. Such a role may even contribute to temporally extended interactions between the two currents involving maintenance or reactivation of traces of cortical activity spanning from minutes to perhaps as much as months (Buzsaki, 1996; Squire and Cohen, 1982; Zola-Morgan and Squire, 1990; Winocur, 1990; see also Moll and Miikulainen, 1997; and further "contextual capture" in Sections $5 \mathrm{~d}$ and e, below). The several functions thus suggested for the countercurrent organization of cortical connectivity may be allocated to different cortical layers, and may utilize different sets or combinations of local interneurons. In any case they operate in the setting of yet another distinctive cortical property, namely its sparsity of point-to-point connectivity.
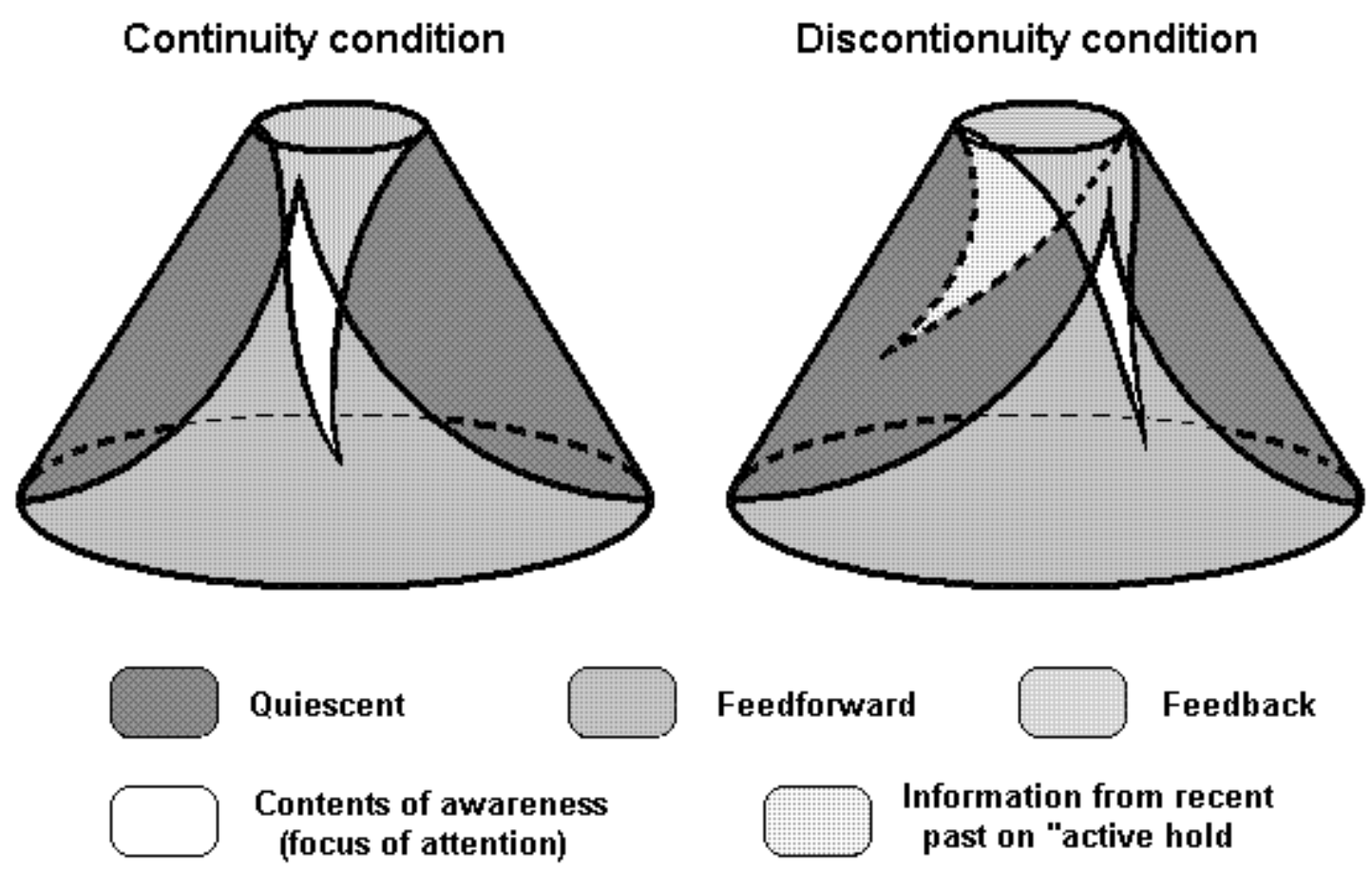

\section{Feedforward}

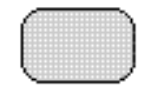

Feedback

\section{Information from recent past on "active hold}

Fig. 4 - Highly schematic depiction of the functional partitioning the "epistemic space" of the cortical solid into "compartments" by activity in the two streams of the countercurrent and their overlap. In the "continuity condition" described in the text there are four such compartments, while the discontinuity condition adds a fifth in the form of information temporarily held in an "overflow buffer." 


\section{$5 d$. The sparsity of cortical connectivity and cascade convergence}

Connectivity between any two pyramidal cells is exceedingly sparse, and may amount to no more than one synaptic contact in a thousand even among local neighbors in the tissue (Braitenberg and Schütz, 1991; see also Douglas and Martin, 1991; Young et al., 1995, fig. 1). The same is true for the proportion of synapses contributed to a given cortical area by one of its close or distant neighbors, which rarely exceeds 5\% (Young, 2000 and references therein). Functionally these circumstances are reflected in the rarity with which cross-correlation methods identify pairs of interconnected cortical cells (Bullier, 1994, $p$. 116).

The sparsity of point-to-point connectivity is a necessary concomitant of the ubiquity of inter-areal connectivity. If the latter is to accomplish convergence onto single cortical cells of multiple sources of convergent afference, no single source of afference must dominate a cell's dendritic tree. This connective sparsity erects a significant barrier to the intracortical propagation of activity originating in any single source of cortical excitation (the excitatory nature of the vast majority of cortical synapses notwithstanding). It appears, nevertheless, to be a general property of cortical tissue, which highlights this structural feature of the neocortex as a fundamental clue to its basic mode of operation. Sparse point-to-point connectivity carries the principle of contextual embedding to its ultimate limit of spatial resolution by vesting it in convergence from multiple sources onto the dendritic surface of individual pyramidal cells. The barrier to propagation of activity is overcome when activity from multiple sources converges onto a given dendritic tree. That is, centripetal convergence from multiple cortical areas, supported by the "cascade" action of multiply staggered inter-areal countercurrent relays (see Figure $3 b$ ), is the normal condition of cortical activation (see also Lamme and Roelfsema, 2000). The result in terms of memory storage is that only cortical loci activated by a sufficiently broad set of bi-directional afferents make a lasting addition to personal history. Convergence from this set of sources - the "connective context set" - ensures the contextual coherence of the storage process, which may be called "contextual capture".

Current knowledge of the long-term consequences for cortical excitability of inter-areal and translaminar interactions does not as yet suffice for more detailed specification of synaptic mechanisms of long-term storage. However, as already noted, laminar segregation of feedforward and feedback connectivity in the cortical sheet ensures that activity in either of the countercurrent streams occurs with some degree of independence (Robert, 1999; see also Douglas and Martin, 1991). Moreover, the interface between activation patterns in the two streams excludes no part of the cortex (see Figure 4; section $5 \mathrm{f}$ below; and Supèr et al., 2001; Ahissar and Hochstein, 1997; Lamme and Roelfsema, 2000; Liang et al., 2000). Since their interaction is continuously ongoing, and the feedback stream relates to activity belonging to the immediate past of the feedforward stream, their interaction amounts to a form of "time shear." This may extend to cortex as a whole the applicability of a "trace learning" paradigm for the acquisition of perceptual invariants (Wallis, 1994; Wallis and Rolls, 1997; Rolls and Milward 2000; Rolls 2000, pp. 615-618).

Another hint concerning storage mechanisms comes from noting the potential utility of "decremental storage" (Sokolov, 1963; Sachs, 1967; Desimone et al., 1994). In a setting pervaded by local inhibitory interneurons a storage process that decreases the excitability of committed synapses will release a local 
neighborhood around storing tissue from the inhibitory influence that was exerted on that neighborhood by the activity of the storing tissue prior to its loss of excitability (Sachs, 1967). Such "marginal sensitization" effects can be seen fleetingly at the level of single cells even in primary sensory areas (Müller et al. 1999) and with longer time-course in perception, notably in perceptual adaptation after-effects (see Durgin 1995, and references therein). At the level of permanent rather than temporary storage such sensitization would promote the progressive (and obligatory) sequential consumption of tissue for memory storage (see Section 3, above) by increasing the probability that tissue at the margins of storing tissue will be the next target of storage. On maps where time receives explicit spatial representation this would generate "lifetime isochronology contours", the existence of which in frontolimbic cortex (see Section 6, below) might be explored with modern imaging methods. More generally, decremental storage provides a dynamic mechanism of cumulative change that acts by "containing" (impeding the propagation of) activity patterns falling within the contextual boundaries of prior personal history, while leaving active those aspects of current activity not represented in prior history ("escape from containment").

\section{5e. Time and epistemic change}

In a contextually organized system the dimension of time will assume a somewhat special status, even though treated on a par with any other variable - a dimension of information among others (Michon and Jackson, 1985). The continuous passage of time ensures that every item or episode of experience, irrespective of any other content or characteristic, will have an entry on the time dimension in the form of time of occurence (or acquisition). The passage of time in otherwise unchanging circumstances provides a baseline condition for the expansion of personal history, underpinning the temporal (historical) aspect of its organization.

Beyond this baseline condition, the cumulative growth of personal history will depend upon a spectrum of strategies for contextual capture predicated upon the extent to which the current content of the focus of attention (sole gate to storage in personal history) is coherent or discrepant with what preceded it. The need for different strategies follows from the spatial organization of the epistemic space outlined in section $5 \mathrm{~b}$ above, in which connective path-length defines the similarity metric of the space. Dissimilar contents are represented far apart while similar ones are represented in spatial proximity within that space (cf. Landauer and Dumais, 1997). Thus, when the content of ongoing experience is in substantial contextual continuity or coherence with what preceded it ("continuity condition", Figure 4), the logistics of its addition to prior history are highly constrained by already active contextual relations. Global contexts will be identical, and discrepant information at lower levels of contextual subsumption will activate locations close to already active ones. The new information will be within reach of the "difference gradient" of contextual embedding extending from already activated patterns and their lingering traces, and will be stored in proximity to these.

Matters stand quite differently in the case of substantial departures in content ("discontinuity condition", Figure 4). The greater the discrepancy or discontinuity in content, the farther afield in epistemic space will potential contextual matches tend to be located. Moreover, the likelihood that they will be encountered in a 
currently activated state is small. Since the needed points of contact with prior history are unavailable in activated form, and storage is contingent on substantial convergent activation, few strategies of coherent storage are available except that of putting the discrepant contents on "active hold" in an "overflow buffer" from which the needed connections may then be established over time, retrospectively and opportunistically.

The ever changing nature of ongoing experience provides an ideal resource for the purpose of retrospective contextual capture of buffered information: the natural variance in ongoing experience of necessity will activate now one, now another portion of prior history over time, and will thus make these accessible in active form to information placed on "active hold." To the extent that ongoing experience happens to activate relevant portions of prior history, buffered information comes within reach of contextual assimilation through countercurrent interaction in a manner similar to that already described for experience in the "continuity condition." No strategy, of course, can provide contextual matches where none exist, as indicated by poor memory performance for truly bizarre information (Worthen and Wood, 2001). The same interaction with the variance supplied by ongoing experience will prevent the integration/ storage process from "getting stuck" in local minima.

The "on hold" strategy also allows economy in the integration process across fluctuations in attentional demand generated by the pressures of living: just as buffered information at the high end of the system sits in readiness to interact with activated contexts, so information held in perceptual stores at the "low" end of the system (Schacter, 1994) provide temporary bridges to subsequent events. Either may come "unbidden to mind" once on-line demands wane. The same relaxation of "attentional load" may permit alternative interpretations of events to be deliberately explored, or an occurrence registered in temporary perceptual storage may be retrospectively retrieved when a new context changes its potential value or significance.

Contextual capture thus provides an interface between at least four components processes: sensory activation carrying information about states of the world; traces of prior sensory events in perceptual storage supplying "bridgeheads" for post-hoc interaction with feedback activity; goals, purposes and "task demands" of the organism defined over its past history; and the contents of a storage buffer at the high end of the system. The rich possibilities for highly variable interactions between these sources would seem to go some way towards explaining the liveliness and diversity of the contents of our consciousness (focal attention or awareness). In fact, much of the everyday happenstance of wandering thoughts, things coming unbidden to mind, rehearsal, retrospection and fantasy may instantiate the fluid interaction between feedforward and feedback activity playing across the layers of the cortical solid during the process of contextual capture. Each such instance, of whatever provenience, supplies another opportunity to form deliberate or tacit contextual linkages between information "on hold" and prior history. Apparently an economy-principle governs time-sharing of the focus of attention between external demands and retrospective contextual assimilation. The process may extend beyond our waking hours into dreaming (Buzsaki, 1996; Stickgold et al., 1999; Stickgold et al., 2000; Graves et al., 2001).

The greater the departure in content between current experience and what preceded it, the greater the 
utility of a strategy which consigns uncontained information to an active memory buffer (cf. Wallenstein et al., 1998) while ever-present changes in ongoing experience supply probes or "reminders" for determining the place of the escaped information with respect to prior knowledge by centripetal convergence. The likelihood of adding further substantial relations by waiting further diminishes with every completed contextual tie-in between information "on hold" and prior history mediated by the occasional relevance of new ongoing experience. That is, the process is one of diminishing returns, and this in turn means that the need for storage of escaped information in a memory buffer is a temporary one.

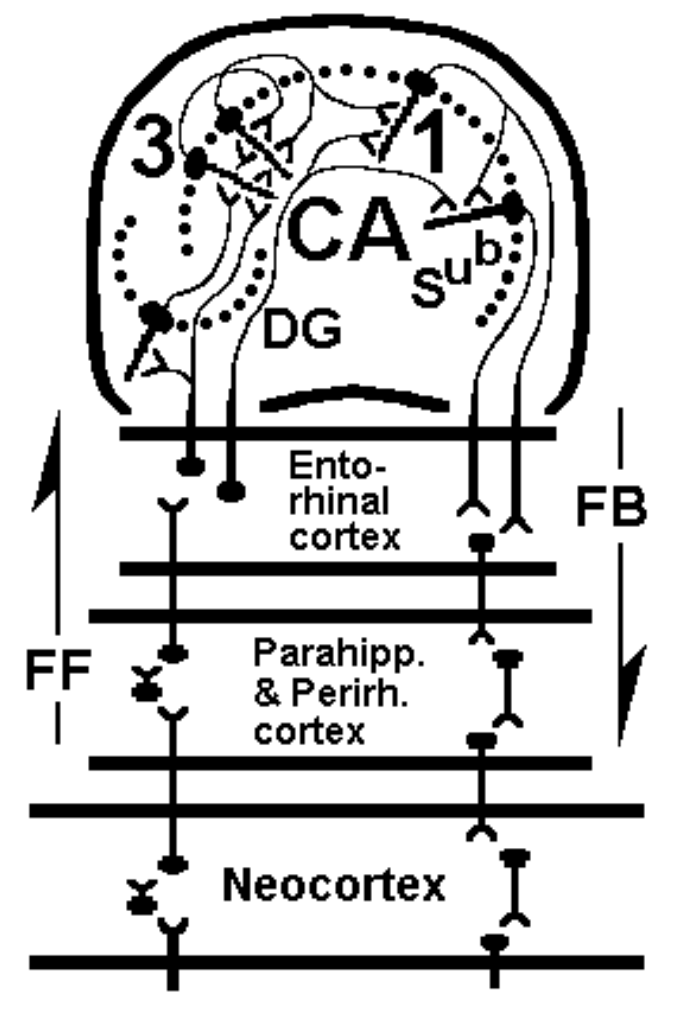

Fig. 5 - Highly schematic depiction of the place of the hippocampal formation in relation to the laminar basis of the cortical countercurrent system.

CA: "Cornu Ammonis", with fields CA3 and

CA1 indicated by numerals.

DG: dentale gyrus;

Sub.: subiculum.

FF and FB: feedforward and feedback connections, respectively.

\section{5f. The hippocampal hinge of the countercurrent system.}

A suitable "overflow buffer" is available in exactly the right place according to the scheme outlined in the foregoing, namely the complex of structures included in the hippocampal formation at the high end of the neural solid (see Figure 1). Anatomically, it forms a "hinge" of the countercurrent system, as schematically illustrated in Figure 5. Neocortical feedforward connections are converted to neocortical feedback connections through a connective loop traversing the parahippocampal and perirhinal cortices, the entorhinal cortex, dentate gyrus, CA fields, and subiculum back to the entorhinal cortex and the neocortex (Witter et al., 1986; Suzuki and Amaral 1994; see also Treves and Rolls, 1994; Buzsaki, 1996; and Figure 5). These connections put "old cortex" at the high end of a vastly larger neocortical pyramid structure.

There its old memory function allows events in neocortex arriving on feedforward pathways to interact with 
earlier and later neocortical events through return feedback pathways, provided they fall within the temporal limits of hippocampal memory. There appears to be a gradient in this regard within the hippocampal formation itself, lengthening as one proceeds from the hippocampus proper towards neocortex (Haist et al., 2001). The unique memory role of this graded storage buffer presumably derives from its position at the high end of the cortical pyramid, that is, at the most global level of contextual subsumption.

Hippocampal formation input to neocortex lies at the origin of the countercurrent feedback cascade, leaving no part of cortex beyond its potential reach. As already noted in section $5 \mathrm{c}$ above, propagation along the feedback pathway is no mere hypothetical possibility, and its influence extends all the way to the primary sensory areas. The global nature of hippocampal influence is reflected in the profound symptoms of its bilateral loss (Scoville and Milner, 1957; Milner, 1966; Zola-Morgan and Squire, 1990; Cipolotti et al., 2001). From the present perspective these symptoms illustrate not only a general dependence of neocortical memory on the countercurrent system, but more specifically its dependence on the contextual mode of cortical storage. The connective position of the hippocampal formation gives it a key role in this regard, by supplying nodes of contextual subsumption at the highest, most global level of the cortical pyramid.

The limited temporal extent of hippocampal formation memory storage has an important consequence for the nature and composition of personal history. If on-going experience does not activate prior contexts relevant to the integration of a given buffered item/episode within some substantial but presumably asymptoting time - say because of a new-found and all-consuming preoccupation - then that buffered item/episode will be effectively lost from the buffer and thus from the possibility of integration with personal history. This means that the content of ongoing experience, a content affected by current interests and concerns, in combination with the buffer's (tuned and asymptotic) temporal storage window performs a dynamic filtering function on what comes to accrete in personal history.

Moreover, memory contents appear to undergo some incremental strengthening of their neural embodiment whenever and to the extent that they are activated through contextual relevance to on-going experience (Sara, 2000; Nader et al., 2000). This means that however durable the synaptic changes in which personal history is stored, the resulting history is never a finally settled affair, but subject to continual passive change through the shifting relatedness of the contents of on-going experience to prior history. This may help explain the vagueness and length of estimates for the time course of "memory consolidation" in humans (Milner, 1966; Squire and Cohen, 1982; Haist et al., 2001; see also Cipolotti et al., 2001) and animals (Zola-Morgan and Squire, 1990; Winocur, 1990). On the present account the storage process is never final, independently of the limited size of the temporal window of hippocampal memory. In fact, the concept of "consolidation" conceived as a process with regular time course hardly applies to it.

It is worth re-emphasizing, finally, that the neural conditions for focal attention or awareness are identical to those for contextual capture, and personal history is essentially a digest of focal experience (cf. Morris and Frey, 1997; Moscovitch 1994; see also Elul and Marchiafava, 1964; Sachs, 1967). Information outside 
of awareness plays a marginal role in its genesis at best (Druckman and Bjork, 1992; Shanks and St John, 1994). This is born out by our contrasting reactions to being confronted with information stored with and without awareness. Items from personal history on being recalled hardly evoke surprise. Surprise is evoked, however, by becoming aware of the presence of an adaptation after-effect. Ordinary usage makes a sound distinction between the two in reserving the term "memory" for the former (see also Metcalfe et al., 1994). The implication is that "we" (i.e. the entity subject to surprise or the lack of it in a given case) must be defined over the entirety of our personal history, which forms a permanent but tacit background to focal awareness.

\section{Biassing storage to serve the storer, or why personal history has to be personal}

The frontolimbic cortex is the contextual high end of the cortical map system. The transition from separate sensory domains to the superordinate frontolimbic domain takes place around levels where the receptotopic organization of each system is being replaced by a more global functional order. "Receptive fields" begin to encompass something approaching the entire receptor array of the modality in question (Van Essen, 1985; Tovee et al., 1994; Oram and Perrett, 1994b; Ito et al., 1995), and unit responses start reflecting high-level informational invariants (Gross et al., 1972; Fuster, 1980; Fuster et al., 1985; Tovee et al., 1994; Wallis and Rolls, 1997; Rolls and Milward, 2000; Rolls, 2000; Kreiman et al., 2000; Miller and Cohen, 2001; Wallis et al., 2001; Freedman et al., 2001). At this level the utility of devoting the two dimensions of a cortical area to the representation of receptor topography (Schwartz, 1984) has been exhausted, leaving room for explicit representation of other parameters, including time, in the spatial layout of a cortical area (see Section $5 \mathrm{~d}$, above). Frontolimbic data formats will therefore differ from those of posterior cortex in significant ways (see, e.g., Wilson and McNaughton 1993; Maunsell 1995, p. 768; Martina et al., 2001; Felleman and Van Essen 1991, p. 37). This difference is plausibly reflected in contrasting contributions to personal history by "posterior" and frontolimbic cortex, respectively. The former has its functional focus in perceptual learning (Rolls and Milward, 2000) with a semantic memory bias (Martin et al., 1995) while the latter emphasizes superordinate relations of subject-centered state variables and causality (see below), with an episodic memory bias (Fletcher et al., 1997).

The frontolimbic cortex is also the domain featuring the full range of motivational, hedonic, state-, task-, and goal-related biasses (Jacobsen, 1935; Nauta, 1971; Fuster, 1980; Watanabe, 1996; Drevets and Raichle, 1994; Wallis and Rolls, 1997; Price, 1999; Asaad et al., 2000; Rolls, 2000; Liu et al., 2000; Miller and Cohen, 2001; Tanji and Hoshi, 2001). The reason for the frontolimbic mix of high level information and motivational bias has a natural interpretation from the point of view of the destiny of its output. The final and highest output of the forebrain does not proceed either from motor cortex or frontal eye fields, areas which in the cortical map system occupy an intermediate, sensori-motor level of organization (see Figure 1, and Young, 1993). Rather, the end results of cortical activity proceed directly from the frontolimbic regions (via pathways such as fornix, stria terminalis and frontal efferents) to the hypothalamus and a paramedian midbrain limbic region (Nauta, 1971) where they help determine the organism's molar 
behavior (Swanson, 2000). Nothing less than the ultimate interpretive powers of the brain suffice when it comes to the moment-to-moment determination of the actual behavioral choices in life. Hence the presence of an inextricably mixed representation of epistemic abstraction and motivational bias in frontolimbic cortex, the ultimate waystation to the hypothalamus.

Global context and motivational bias are intimately related. From the point of view of the cortical map system as a whole, tasks such as object identification (Olson, 2001) are but a part of the fundamental task of assessing the significance of the current situation as a whole in light of the organism's needs and goals, both immediate and long-term. The meaning of a situation - any situation - has no definition apart from the nature and purposes of the organism finding itself in that situation. Once the characteristics of the agent are given, scene interpretation translates into action plans. The world whose significance the brain is assessing is the arena on which the consequences of its interpretations and choices will be experienced. This is no neutral matter, nor should it be, and the countercurrent organization of cortex provides the means for "inserting", as it were, the personal subject-centered "viewpoint" into the structure of the world. The origin of the feedforward current in sensory areas mapping current states of the world in high-resolution detail supplies the objective or veridical pole of this process ("the (sensory) world"), while frontolombic feedback with its motivational, state- and goal-related functions supplies the subjective or personal pole ("the (motivated) person"). Their interaction brings the needs, motives, goals and purposes of the individual to bear on circumstances such as they exist in the world, and drives the reconciliation of these two domains in instances where they turn out to be discrepant or in conflict. The interaction yields the contents of awareness in the form of observer-centered personal experience with its motivational, hedonic and goal-related biasses as its short-term ("on-line") result, and personal history in the form of the record of the fate of an individual's motivated acts in the world over time as its long term residue.

\section{Conclusions}

In the foregoing the concept "escape from contextual containment" has been used as an informal shorthand for the key dynamic agency in the accretion of personal history. Might there be a more precise definition of the conditions that drive the expansion of personal history by exceeding its prior bounds? "Novelty", "incomprehension", "consequentiality" (see below) are all such conditions, but they resist inclusion under any more telling superordinate category than an overly broad one such as "mattering." This is so for the basic reason that "what matters" to a mammal, and especially to a large-brained mammal, is defined by a vast multidimensional system of longitudinally accumulated and contextually integrated experience which is far too rich and multifacetted to be reduced to any single generative principle, and especially not one ignoring the motivational complexities of evolved creatures. This, according to the present perspective, is the same as saying that they are equipped with a neocortex.

Ultimately any mismatch between the world as it comes to us and the world as we have come to expect or desire it on the basis of our experience is a high priority candidate for primary agency in driving the cumulative expansion of personal history. That would include any circumstance harboring potential consequences, even remote ones, for our lot in life, extending to whomever and whatever we hold dear 
and care about. In this sense, then, "consequentiality" is the central dynamic agency for the accretion of personal history. This is what would be expected of evolved organisms if they had access to the kind of sophisticated inductive knowledge that allowed them to sense the implications of their current situation in the light of their past experience. The above account suggests that it may not be possible to equip them with such a capacity short of the laminarly segregated and multiply connected countercurrent organization evolved in the distinctively mammalian structure of the cortex. The great mammalian radiation would accordingly be a vast biological experiment with the possibilities latent in a globally connected epistemic space designed for the inductive acquisition of higher order knowledge on the basis of cumulative recording and contextual ordering of lifetime memory.

Needless to say such a function is bound to have an impact on temporal horizons of external mortality, whose statistics, it must be remembered, are determined only partially by mortality attributable to causes in the physical and biotic environment. The remainder is accounted for by factors in the conspecific environment. These factors assume particular prominence in social species (Humphrey, 1976; Byrne and Whiten 1988), and those with elaborate culture above all. Besides humans these include hominoids (Whiten et al., 1999) and some cetaceans (Noad et al., 2000; Payne, 2000; Whitehead, 1998), forms which also carry large cortices. The informational resources of these large cortices, deployed for warding off external mortality, would close the causal loop of life history determination by ensuring the extended life-span needed for growing a large body, the same life-span over which perpetual memory recording induces the sophisticated knowledge needed to insure the survival of that body in an uncertain world. Should the present proposal concerning the functional organization of that process turn out to have any merit, further specification of the mechanisms involved in the accretion of personal history will be a matter of unravelling the long-term impact on cortical excitability of interaction between "the (sensory) world" and "the (motivated) person" via the two streams of the cortical countercurrent system, a topic of active research whose details are decidedly beyond the scope of the present proposal, conceived as a mere outline of a scheme within which such detail may eventually find a fitting functional context.

\section{Acknowledgements:}

I dedicate this paper to the memory of the late Eugene Sachs, who taught me the basics of the ideas I have developed here. I am also indebted to Stephan Syz for assistance in matters of style, and to Adrian Robert, Maria Ujhelyi, and three anonymous reviewers for helpful comments on matters of content. The author was supported by a grant from the Bank of Sweden Tercentenary Foundation. 


\section{References:}

Abeles M. Corticonics. Neural circuits of the cerebral cortex. Cambridge, UK: Cambridge University Press, 1991.

Aboitiz, F. Evolution of isocortical organization. Cerebral Cortex, 9: 655-661, 1999.

Adelson EH, Simoncelli EP and Freeman WT. Pyramids and Multiscale Representations. In A. Gorea (Ed.), Representations of Vision, pp. 3-16. Cambridge, UK: Cambridge University Press, 1991.

Ahissar M and Hochstein S. Task difficulty and the specificity of perceptual learning. Nature, 387: 401406, 1997.

Allman J M. Evolution of the visual system in the early primates. Progress in Psychobiology and Physiological Psychology, 7: 1-53, 1977.

Allman JM. Evolving Brains. New York: Scientific American Library, 1999.

Asaad WF, Rainer G and Miller EK. Task-specific neural activity in the primate prefrontal cortex. Journal of Neurophysiology, 84: 451-459, 2000.

Banavar JR, Maritan A and Rinaldo A. Size and form in efficient transportation networks. Nature, 399: 130-132, 1999.

Barone P and Joseph JP. Prefrontal cortex and spatial sequencing in macaque monkey. Experimental Brain Research, 78: 447-464, 1989.

Barton RA. Neocortex size and behavioral ecology in primates. Proceedings of the Royal Society, London, Series B, 263: 173-177, 1996.

Biegler R, McGregor A, Krebs J R and Healy S D. A larger hippocampus is associated with longer-lasting spatial memory. Proceedings of the National Academy of Sciences (USA), 98: 6941-6944, 2001.

Binford LR. In pursuit of the past. Decoding the archaeological record. New York: Thames and Hudson, 1983.

Bingman VP, Bagnoli $P$, loalè $P$ and Casini G. Behavioral and anatomical studies of the avian hippocampus. Neurology and Neurobiology, 52: 379-394, 1989.

Braitenberg V. Thoughts on the cerebral cortex. Journal of Theoretical Biology, 46: 421-447, 1974.

Braitenberg, V. Anatomical basis for divergence, convergence and integration in the cerebral cortex. Advances in Physiological Science, 16: 411-419, 1981.

Braitenberg V and Schüz A. Anatomy of the cerebral cortex. Statistics and geometry. Berlin: Springer Verlag, 1991.

Braitenberg V. On the texture of brains. Berlin: Springer Verlag, 1977, Ch. 8, pp. 101-122.

Brodmann K. Vergleichende Lokalisationslehre der Großhirnrinde in ihren Prinzipien dargestellt auf Grund des Zellenbaues. Leipzig. Barth, 1909.

Bruce C, Desimone R and Gross C G. Visual properties of neurons in a polysensory area in superior temporal sulcus of the macaque. Journal of Neurophysiology, 46: 369-384, 1981. 
Bullier J. Introduction: Searching for the operational principle of cortex. In: Albowitz et al. (Eds), (1994). Structural and functional organization of the neocortex. Berlin: Springer Verlag, 1994, p. 116.

Burgess C, Livesay K, and Lund K. Explorations in context space: Words, sentences, discourse. Discourse Processes, 25: 211-257, 1998

Burgess $C$ and Lund K. The dynamics of meaning in memory. In E. Dietrich and A.B. Markman (Eds.), Cognitive dynamics: conceptual and representational change in humans and machines. Lawrence Erlbaum, 2000.

Burns GAPC and Young MP. Analysis of the connectional organization of neural systems associated with the hippocampus in rats. Philosophical Transactions of the Royal Society, London, Series B, 355: 55-70, 2000.

Buzsaki G. The hippocampo-neocortical dialogue. Cerebral Cortex, 6: 81-92, 1996.

Byrne RW and Whiten A. Machiavellian Intelligence. Social expertise and the evolution of intellect in monkeys, apes and humans. Oxford: Clarendon Press, 1988.

Callaway EM. Local circuits in the primary visual cortex of the macaque monkey. Annual Review of Neuroscience, 21: 47-74, 1998.

Charnov EL. Life history invariants. Some explorations of symmetry in evolutionary ecology. Oxford: Oxford University Press, 1993.

Cipolotti L, Shallice T, Chan D, Fox N, Scahill R, Harrison G, Stevens J and Rudge P. Long-term retrograde amnesia... the crucial role of the hippocampus. Neuropsychologia, 39: 151-172, 2001.

Clutton-Brock T and Harvey P. Primates, brains and ecology. Journal of Zoology, London, 190: 309-323, 1980.

Craik FIM and Tulving E. Depth of processing and the retention of words in episodic memory. Journal of Experimental Psychology: General, 104: 268-294, 1975.

Creutzfeldt OD. Generality of the functional structure of the neocortex. Naturwissenschaften, 64: 507-517, 1977.

Creuzfeldt OD. The neocortical link: Thoughts of the generality of structure and function of the neocortex. In Brazier MAB and Petsche H (Eds.) Architectonics of the Cerebral Cortex. New York: Raven Press, 1978.

Damasio AR. The brain binds entities and events by multiregional activation from convergence zones. Neural Computation, 1: 123-132, 1989.

Dayan P, Hinton G E, Neal R M and Zemel RS. The Helmholtz machine. Neural Computation, 7: 889-904, 1995.

Deacon TW. Holism and associationism in neuropsychology: An anatomical synthesis. In Perecman E (Ed.), Integrating theory and practice in clinical neuropsychology. Hillsdale, New Jersey: Erlbaum, 1989, pp. 1-47.

Desimone R, Miller E K and Chelazzi L. The interaction of neural systems for attention and memory. In Koch C and Davis J L (Eds.), Large-Scale Neuronal Theories of the Brain. Cambridge MA: MIT Press, 1994, pp. 75-92. 
Devoogd T J, Krebs J R, Healy S D and Purvis A. Relations between song repertoire size and the volume of brain nuclei related to song: comparative evolutionary anaysis amongst oscine birds. Proceedings of the Royal Society, London, Series B, 254: 75-82, 1993.

Douglas RJ and Martin KAC. Opening the grey box. Trends in Neuroscience, 14: 286.293, 1991.

Drevets WC and Raichle ME. PET imaging studies of human emotional disorders. In Gazzaniga M (Ed.). The Cognitive neurosciences. Cambridge, Mass: MIT Press, 1994, pp. 1153-1164.

Druckman D and Bjork RA (Eds.). In the mind's eye: Enhancing human performance. National Research Council, 1992.

Dunbar RIM. Neocortex size and group size in primates: A test of the hypothesis. Journal of Human Evolution, 28: 289-296, 1995.

Durgin $\mathrm{FH}$. Visual aftereffect of texture density contingent on color of frame. Perception and Psychophysics, 58: 207-223, 1995.

Economos AC. Brain-life span conjecture: A re-evaluation of the evidence. Gerontology, 26: 82-89, 1980.

Eisenberg $\mathrm{J}$ and Wilson D. Relative brain size and feeding strategies in chiroptera. Evolution (Lawrence, Kansas), 32: 740-751, 1978.

Elul $\mathrm{R}$ and Marchiafava PL. Accomodation of the eye as related to behavior in the cat. Archives Italiennes de Biologie, 102: 616-644, 1964.

Fahlman SE. The recurrent cascade-correlation architecture. In Lippman RP, Moody JE and Touretzky DS (Eds.), Advances in Neural Information Processing Systems 3. San Mateo, CA: Morgan Kaufmann, 1991, pp. 190-205.

Fahlman SE and Lebiere C (1990). The cascade correlation architecture. In Lippman RP, Moody JE and Touretzky DS (Eds.), Advances in Neural Information Processing Systems 2. San Mateo, CA: Morgan Kaufmann, 1990, pp. 524-532.

Felleman DJ and Van Essen DC. Distributed hierarchical processing in the primate cerebral cortex. Cerebral Cortex, 1: 1-47, 1991.

Finch CE. Longevity, Senescence and the Genome. University of Chicago Press, Chicago, 1990.

Finlay BL and Darlington RB. Linked regularities in the development and evolution of mammalian brains. Science, 268: 1578-1584, 1995.

Fletcher PC, Frith CD and Rugg MD. The functuional neuroanatomy of episodic memory. Trends in Neuroscience, 20: 213-218, 1997.

Freedman DJ, Riesenhuber M, Poggio T, and Miller EK. Categorical representation of visual stimuli in the primate prefrontal cortex. Science, 291: 312-316, 2001.

Friston K. Functional integration and inference in the brain. Progress in Neurobiology, 68: 113-143, 2002.

Fuster JM. The prefrontal cortex. New York: RAVEN Press, 1980.

Fuster JM, Bauer RH and Jervey JP. Cellular discharge in the dorsolateral prefrontal cortex of the monkey in cognitive tasks. Experimental Neurology, 77: 679-694, 1985. 
Graves I, Pack A and Abel T. Sleep and memory: a molecular perspective. Trends in Neurosciences, 24: 237-243, 2001.

Gross $\mathrm{CH}$, Rocha-Miranda CE and Bender DB. Visual properties of neurons in inferotemporal cortex of the macaque. Journal of Neurophysiology, 35: 96-111, 1972.

Grossberg S. Competitive learning: From interactive activation to adaptive resonance. Cognitive Science, 11: 23-63, 1987.

Grossberg S and Merrill JWL. The Hippocampus and Cerebellum in adaptively timed learning, recognition, and movement. Journal of Cognitive Neuroscience, 8: 257-277, 1996.

Haft M. Robust "topological" codes by keeping control of internal redundancy. Physical Review Letters, 81: 4016-4019, 1998.

Haist F, Gore JB and Mao H. Consolidation of human memory over decades revealed by functional magnetic resonance imaging. Nature Neuroscience, 4: 1139-1145, 2001.

Hakeem A, Rodriguez SG, Jones M and Allman J. Brain and lifespan in primates. In: Birren JE and Schaie KW (Eds). Handbook of the Psychology of Aging. New York: Academic Press, 1996.

Hampton RR, Sherry DF, Shettleworth SJ, Khurgel M. Hippocampal volume and food storing behavior are related in parids. Brain, Behavior and Evolution, 45: 54-61, 1995.

Harvey P H, Clutton-Brock T H and Mace G M. Proceedings of the National Academy of Sciences, U.S.A., 77: 4387, 1980

Harvey PH and Krebs JR. Comparing brains. Science, 249: 140-146, 1990.

Healy SD, Gwinner E and Krebs JR. Hippocampal volume in migratory and non migratory warblers:

effects of age and experience. Behavioural Brain Research, 81: 61-68, 1996.

Hilgetag C-C, O'Neill MA and Young MP. Indeterminate organization of the visual system. Science, 271: 776-777, 1996.

Hofman MA. Encephalization and the evolution of longevity in mammals. Journal of Evolutionary Biology, 6: 209-227, 1993.

Hopfield, JJ. Neural networks and physical systems with emergent collective computational abilities. Proceedings of the National Academy of Sciences, USA, 79: 2554-2558, 1982.

Humphrey NK. The social function of intellect. In Bateson PPG and Hinde RA (Eds.). Growing Points in Ethology. Cambridge: Cambridge University Press, 1976, pp. 303-317.

Ito M, Tamura H, Fujita I, and Tanaka K. Size and position invariance of neural responses in monkey inferotemporal cortex. Journal of Neurophysiology, 73: 218226, 1995.

Jacobsen CF. Functions of the frontal association area in primates. Archives of Neurology and Psychiatry, 33: 558, 1935.

Jerison H. Evolution of the brain and intelligence. New York: Academic Press, 1973.

Joffe TH and Dunbar RIM. Visual and socio-cognitive information processing in primate brain evolution. Proceeding of the Royal Society of London, Series B, 264: 1303-1307, 1997. 
Jones EG and Powell TPS. An anatomical study of converging sensory pathways within the cerebral cortex of the monkey. Brain, 93: 793-820, 1970.

Kaas, J. H. The evolution of isocortex. Brain, behavior and Evolution, 46: 187-196, 1995.

Kawato M, Hayakawa $\mathrm{H}$ and Inui T. A forward-inverse optics model of reciprocal connections between visual cortical areas. Network, 4: 415-422, 1993.

Kirkwood TBL and Holliday R (1986). Ageing as a consequence of natural selection. In Bittles AH and Collind KJ (Eds.) The Biology of Human Ageing. Cambridge: Cambridge University Press, 1986, pp. 1-16.

Kirkwood TBL and Rose MR. Evolution of senescence: Late survival sacrificed for reproduction. Philosophical Transactions of the Royal Society, London, Series B, 332: 15-24, 1991.

Kleiber M. Body size and metabolism. Hilgardia, 6: 315-353, 1932.

Kleiber M. The Fire of Life. An Introduction to Animal Energetics. New York: John Wiley and Sons, 1961.

Krebs JR. Food-storing birds: Adaptive specialization in brain and behaviour? Philosophical Transactions of the Royal Society, London Series B, 329: 153-160, 1990.

Kreiman G, Koch C and Fried I. Category-specific visual responses of single neurons in the human medial temporal lobe. Nature Neuroscience, 3: 946-953, 2000.

Lamme VAF and Spekreijse $\mathrm{H}$. Modulations of primary visual cortex activity represent attentive and conscious scene perception. Frontiers in Bioscience, 5: 232-243, 2000.

Lamme VAF and Roelfsema PR. The distinct modes of vision offered by feedforward and recurrent processing. Trends in Neuroscience, 23: 571-579, 2000.

Landauer TK and Dumais ST. A solution to Plato's problem: The latent semantic analysis theory of acquisition, induction and representation of knowledge. Psychological Review, 104: 211-240, 1997.

Landauer TK, Foltz PW and Laham D. Introduction to latent semantic analysis. Discourse Processes, 25 : 259-284, 1998.

Lashley KS (1951). The problem of serial order in behavior. In Jeffress LA (Ed.), Cerebral Mechanisms and Behavior. New York: Wiley, 1951, pp. 112-136.

Lefebvre L, Whittle P, Lascaris E and Finkelstein A. Feeding innovations and forebrain size in birds. Animal Behaviour, 53: 549-560, 1997.

Liang H, Ding M, Nakamura R and Bressler SL. Causal influences in primate cerebral cortex during visual pattern discrimination. NeuroReport, 11: 2875-2880, 2000.

Liu Z, Murray EA and Richmond BJ. Learning motivational signifiance of visual cues for reward schedules requires rhinal cortex. Nature Neuroscience, 3: 1307-1315, 2000.

Lindstedt S and Calder W. Body size and longevity in birds. Condor, 78: 91-94, 1976.

Livingstone $M$ and Hubel D. Segregation of form, color, movement, and depth:anatomy, physiology, and perception. Science, 240: 740-749, 1988.

Lund, K., \& Burgess, C. (1996). Producing high-dimensional semantic spaces from lexical co-occurrence. Behavior Research Methods, Instruments, and Computers, 28: 203-208. 
Mace GM, Harvey PH and Clutton-Brock TH. Brain size and ecology in small mammals. Journal of Zoology, London, 193: 333-254, 1981.

Marin-Padilla, M. Cajal-Retzius cells and the development of the neocortex. Trends in Neurosciences, 21: 64-71, 1998.

Martin A, Haxby JV, Lalonde FM, Wiggs CL and Underleider LG. Discrete cortical regions associated with knowledge of color and knowledge of action. Science, 270: 102-105, 1995.

Martina M, Royer S and Paré D. Propagation of neocortical inputs in the perirhinal cortex. Journal of Neuroscience, 21: 2878-2888, 2001.

Maunsell JHR. The brain's visual world: representation of visual targets in cerebral cortex. Science, 270: 764-769, 1995.

McCloskey M and Cohen NJ. Catastrophic interference in connectionist networks: The sequential learning problem. The Psychology of Leraning and Motivation, 24: 109-165, 1989.

Mcllwain J. Point images in the visual system: new interest in an old idea. Trends in Neuroscience, 9: 354358, 1986.

Medawar PB. An Unsolved Problem in Biology. H.K.Lewis, London, 1952.

Metcalfe J, Mencl WE and Cottrell GW. Cognitive binding. In: Schacter DL and Tulving E (Eds.), Memory Systems 1994. Cambridge, MA: MIT Press, 1994, pp. 369-394.

Michon JA and Jackson JL. Introduction: The psychology of time. In: J A Michon and JL Jackson (Eds.), Time, Mind and Behavior. New York: Springer, 1985, pp. 2-17.

Mignard M and Malpelli JG. Paths of information flow through visual cortex. Science, 251: 1249-1251, 1991.

Miller EK and Cohen JD. An integrative theory of prefrontal cortex function. Annual Review of Neuroscience, 24: 167-202, 2001.

Milner B. Amnesia following operations on the temporal lobes. In Witty CWM and Zangwill OL (Eds.), Amnesia. London: Butterworth, 1966, pp. 109-133.

Moll $\mathrm{M}$ and Miikkulainen R. Convergence-zone episodic memory: Analysis and simulations. Neural Networks, 10: 1017-36, 1997.

Morris RGM and Frey U. Hippocampal synsptic plasticity: role in spatial learning or the automatic recording of attended excperience? Philosophical Transactions of the Royal Society, London, Series B., 352: 1489-1503, 1997.

Moscovitch, M. Memory and working with memory: Evaluation of a component process model and comparison with other models. In Schacter DL and Tulving E (Eds.). Memory Systems 1994. Cambridge, MA: MIT Press, 1994, pp. 269-310.

Müller JR, Metha AB, Krauskopf J and Lennie P. Rapid adaptation in visual cortex to the structure of images. Science, 285: 1405-1408, 1999.

Nader K, Schafe GE and LeDoux JE. Fear memories require protein synthesis in the amygdala for reconsolidation after retrieval. Nature, 406: 722-726, 2000. 
Nauta WJH. The problem of the frontal lobe: A reinterpretation. Journal of Psychiatric Research, 8: 167$187,1971$.

Nieuwenhys, R. The neocortex: An overview of its evolutionary development, structural organization and synaptology. Anatomy and Embryology, 190: 307-337, 1994.

Noad MJ, Cato DH, Bryden MM, Jenner M-N and Jenner KCS. Cultural revolution in whale song. Nature, 408: 538, 2000.

Olson CR. Object-based vision and attention in primates. Current Opinion in Neurobiology, 11: 171-179, 2001.

Oram, M and Perrett, D. Responses of anterior superior temporal polysensory (STPa) neurons to "biological motion" stimuli. Journal of Cognitive Neuroscience, 6: 99-116, 1994a.

Oram, MW and Perrett DI. Modeling visual recognition from neurobiological constraints. Neural Networks, 7: 945972, 1994b.

Passingham RE. The brain and intelligence. Brain Behavior and Evolution, 11: 1-15, 1975.

Patel SJ, Clayton NS \& Krebs JR. Spatial learning induces neurogenesis in the avian brain. Behavioural Brain Research, 89: 115-128, 1997.

Payne BR, Lomber SG. A method to assess the functional impact of cerebral connections on target populations of neurons. Journal of Neuroscience Methods, 86: 195-208, 1999.

Payne, K. The progressively changing songs of humpback whales: A window on the creative process in a wild animal. In NL Wallin, B Merker and S Brown, eds. The Origins of Music. The MIT Press, Cambridge, MA, 2000, pp. 135-150.

Pirlot P and Stephan H. Encephalization in the chiroptera. Canadian Journal of Zoology, 48: 433-444, 1970.

Price JL. Prefrontal cortical networks related to visceral function and mood. Annals of the New York Academy of Sciences, 877: 383-396, 1999.

Rao RPN and Ballard DH. Dynamic model of visual recognition predicts neural response properties in the visual cortex. Neural Computation, 9: 805-47, 1997.

Robert A. Lamination and within-area integration in the neocortex. Doctoral dissertation, Cognitive Science, University of California, San Diego, 1999.

Rockel AJ, Hiorns RW and Powell TPS. The basic uniformity in structure of the neocortex. Brain, 103: 221-244, 1980.

Rockland KS and Pandya DN. Laminar origins and terminations of cortical connections to the occipital lobe in the rhesus monkey. Brain Research, 179: 3-20, 1979.

Rolls ET. Memory systems in the brain. Annual Review of Psychology, 51: 599-630, 2000.

Rolls ET and Milward T. A model of invariant object recognition in the visual system: learning rules, activation functions, lateral inhibition, and information-based performance measures. Neural Computation, 12: 2547-2572, 2000. 
Sacher GA. Relationship of lifespan to brain weight and body weight in mammals. In Wolstenholme GEW and O'Connor M (Eds.), C.I.B.A. Foundation Symposium on the Lifespan of Animals. Boston, MA: Little and Brown, 1959, pp. 115-133.

Sacher GA. Evaluation of the entropy and information terms governing mammalian longevity. In Cutler RG (Ed.) Interdisciplinary Topics in Gerontology, vol 9. Karger, Basel, 1976, pp. 69-83.

Sachs E. Dissociation of learning in rats and its similarities to dissociative states in man. In Zubin $\mathrm{J}$ and Hunt H (Eds.), Comparative Psychopathology: Animal and Human. New York: Grune and Stratton, 1967, pp. 249-304.

Salin P-A and Bullier J. Corticocortical connections in the visual system: Structure and function. Physiological Reviews, 75: 107-154, 1995.

Sara SJ. Retrieval and reconsolidation: toward a neurobiology of remembering. Learning and Memory, 7: 73-84, 2000.

Sawaguchi T. Possible involvement of sexual selection in neocortical evolution of monkeys and apes. Folia Primatologica, 68: 95-99, 1997.

Schacter DL. Priming and multiple memory systems: Perceptual mechanisms of implicit memory. In Schacter DL and Tulving E (Eds.). Memory systems 1994. Cambridge, MA: MIT Press, 1994, pp. 233-268.

Schmidhuber J. The Neural Heat Exchanger. In S Amari, L Xu, L Chan, I King and K Leung (Eds), Progress in Neural Information Processing: Proceedings of the Intl. Conference on Neural Information Processing, pp. 194-197. Hongkong: Springer Verlag, 1996.

Schmidt-Nielsen K. Scaling in biology: the consequence of size. Journal of Experimental Zoology, 194: 287-308, 1975.

Schmidt-Nielsen K. Animal Physiology. Cambridge: Cambridge University Press, 1997.

Schwartz EL. Spatial mapping and spatial vision in primate striate and infero-temporal cortex. In Spillman L and Wooten BR (Eds.), Sensory Experience, Adaptation and Perception. Festschrift for Ivo Kohler.

Hillsdale, New Jersey: Lawrence Erlbaum, 1984, pp. 73-104.

Scoville WB and Milner B. Loss of recent memory after after bilateral hippocampal lesions. Journal of Neurology, Neurosurgery and Psychiatry, 20: 11-21, 1957.

Shanks DR and St John MF. Characteristics of dissociable human learning systems. Behavioral and Brain Sciences, 17: 367-447, 1994.

Sokolov EN. Higher nervous functions: The orienting reflex. Annual Review of Physiology, 25: 545-580, 1963.

Somogyi $P$. The order in synaptic circuits of the cerebral cortex: lessons from the hippocampus. In Albowitz B, Albus K, Kuhnt U, Nothdurft H-Ch and Wahle P (Eds.), Structural and functional organization of the neocortex. Springer, Berlin, 1994, pp. 148-161.

Sporns O, Tononi G and Edelman GM. Theoretical neuroanatomy: Relating anatomical and functional connectivity in graphs and cortical connection matrices. Cerebral Cortex, 10: 127-141, 2000.

Squire LR and Cohen N. Remote memory, retrograde amnesia, and the neuropsychology of memory. In Cermak LS (Ed.), Human memory and amnesia. Hillsdale, New Jersey: Erlbaum, 1982, pp. 275-303. 
Stephan $\mathrm{H}$, Nelson $\mathrm{J}$ and Frahm $\mathrm{H}$. Brain size comparison in chiroptera. Zeitschrift fürZoologische Systematik und Evolutionsforschung, 19: 195-222, 1981.

Stephan KE, Hilgetag C-C, Burns GAPC, O'Neill MA, Young MP and Kötter R. Computational analysis of functional connectivity between areas of primate cerebral cortex. Philisophical Transactions of the Royal Society, London, Series B, 355: 111-126, 2000.

Steedman, M. Connectionist sentence processing in perspective. In: Special issue of Cognitive Science on Connectionist Approaches to Sentence Processing, 1999.

Stickgold R, Malia A, Maguire D, Roddenberry, D and O'Connor m. Replaying the game: Hypnagogic images in normals and amnesics. Science, 290: 350-353, 2000.

Stickgold R, Scott L, Rittenhouse C and Hobson JA. Sleep induced changes in associative memory. Journal of Cognitive Neuroscience, 11: 182-193, 1999.

Striedter GF. The telencephalon of tetrapods in evolution. Brain, Behavior and Evolution, 49: 179-213, 1997.

Supèr $\mathrm{H}$, Spekreijse $\mathrm{H}$ and Lamme VAF. A neural correlate of working memory in the monkey primary visual cortex. Science, 293: 120-124, 2001.

Suzuki WA and Amaral DG. Perirhinal and parahippocampal cortices of the macaque monkey: cortical afferents. Journal of Comparative Neurology, 350: 497-533, 1994.

Swanson L W. Cerebral hemisphere regulation of motivated behavior. Brain Research, 886: 113-164, 2000.

Tanji $\mathrm{J}$ and Hoshi E. Behavioral planning in the prefrontal cortex. Current Opinion in Neurobiology, 11: 164-170, 2001.

Teuber, H.-L. The brain and human behavior. In R. Held, H.W. Leibowitz \& H.-L. Teuber (Eds.), Handbook of Sensory Physiology, VIII: Perception, pp. 879-920. Berlin: Springer Verlag, 1978.

Timmermans S, Lefebvre L, Boire D and Basu P. Relative size of the hyperstriatum ventrale is the best predictor of feeding innovation rate in birds. Brain, Behavior and Evolution, 56: 196-203, 2000.

Tomita H, Ohbayashi M, Nakahara K, Hasegawa I and Miyashita Y. Top-down signal originating from the prefrontal cortex for memory retrieval. Nature, 401: 699-703, 1999.

Tononi G, Sporns $\mathrm{O}$ and Edelman GM. A measure for brain complexity: Relating functional segregation and integration in the nervous system. Proceedings of the National Academy of Sciences USA, 91: 50335037, 1994.

Tovee MJ, Rolls ET, and Azzopardi P. Translation invariance in the responses to faces of single neurons in the temporal visual cortical areas of the alert macaque. Journal of Neurophysiology, 72: 10491060, 1994.

Treves A and Rolls ET. A computational analysis of the role of the hippocampus in memory. Hippocampus, 4: 374-391, 1994.

Tucker DM and Desmond RE Jr. Ageing and the plasticity of the self. In Schaie KW and Lawton MP (Eds.), Annual Review of Gerontology and Geriatrics. New York: Springer, 1998, pp. 266-281.

Tulving E. Episodic and semantic memory. In Tulving E and Donaldson W (Eds.), Organization of Memory. New York: Academic Press, 1972, pp. 381-403. 
Ullman S. Sequence seeking and counter streams: A computational model for bidirectional information flow in the visual cortex. Cerebral Cortex, 5: 1-11, 1995.

de Waal F. Chimpanzee politics: Power and sex among apes. New York: Harper, 1982.

Van Essen D. Functional organization of primate visual cortex. In Peters A and Jones EG (Eds.), Cerebral Coprtex, Vol. 3. New York, Plenum, 1985, pp. 259-329.

Volman SF, Grubb TC and Schuett KC. Relative hippocampal volume in relation to food-storing behavior in four species of woodpeckers. Brain, Behavior and Evolution, 49: 110-120, 1997.

Wallenstein GV, Eichenbaum $\mathrm{H}$ and Hasselmo ME. The hippocampus as an associator of discontiguous events. Trends in Neuroscience, 21: 317-323, 1998.

Wallis G. Neural mechanisms underlying processing in the visual areas of the occipital and temporal lobes. Doctoral Thesis, Department of Experimental Psychology, University of Oxford, 1994.

Wallis $\mathrm{G}$ and Rolls ET. Invariant face and object recognition in the visual system. Progress in Neurobiology, 51: 167-194, 1997.

Wallis JD, Anderson KC, and Miller EK. Single neurons in prefrontal cortex encode abstract rules. Nature, 411: 953 - 956, 2001.

Watanabe M. Reward expectancy in primate prefrontal neurons. Nature, 382: 629-632, 1996.

West GB, Brown JH and Engquist BJ. The fourth dimension of life: Fractal geometry and allometric scaling of organisms. Science, 284: 16771679, 1999.

West GB, Brown JH and Engquist BJ. A general model for the origin of allometric scaling laws in biology. Science, 276: 122-126, 1997.

Whitehead H. Cultural selection and genetic diversity in matrilineal whales. Science, 282: 1708-1711, 1998.

Whiten A, Goodall J, McGrew WC, Nishida T, Reynolds V, Sugiyama Y, Tutin CEG, Wrangham RW, and Boesch C. Cultures in Chimpanzees. Nature, 399: 682-685, 1999.

Williams GC. Pleiotropy, natural selection and the evolution of senescence. Evolution, 11: 398-411, 1957.

Wilson MA and McNaughton BL. Dynamics of the hippocampal ensemble code for space. Science, 261: 1055-1058, 1993.

Winocur G. Anterograde and retrograde amnesia in rats with dorsal hippocampal or dorsomedial thalamic lesions. Behavioral Brain Research, 38: 145-154, 1990.

Witkin J. Primate brain and life history. Unpublished doctoral dissertation. Columbia University, New York, 1980.

Witter MP, Room P, Groenewegen HJ and Lohman AHM. Connections of the parahippocampal cortex in the cat. V. Intrinsic connections; comments on input/output connections with the hippocampus. Journal of Comparative Neurology, 252: 78-94, 1986.

Worthen JB and Wood VV. A disruptive effect of bizarreness on memory for relational and contextual details of self-performed and other-performed acts. American Journal of Psychology, 114: 535-546, 2001. 
Young MP. Objective analysis of the topological organization of the primate visual system. Nature, 358: 152-155, 1992.

Young, MP. The organization of neural systems in the primate cerebral cortex. Proceedings of the Royal Society, London, Series B, 252: 13-18, 1993.

Young MP. The architecture of visual cortex and inferential processes in vision. Spatial Vision, 13: 137146, 2000.

Young MP, Scannell JW, O'Neill MA, Hilgetag CC, Burns B and Blakemore C. Non-metric multidimensional scaling in the analysis of neuroanatomical connection data and the organization of the primate cortical visual system. Philosophical Transactions of the Royal Society, London, Series B, 348: 281-308, 1995.

Zeki SM. Uniformity and diversity of structure and function in rhesus monkey prestriate cortex. Journal of Physiology (London), 277: 273-290, 1978.

Zola-Morgan SM and Squire LR. The primate hippocampal formation: Evidence for a time-limited role in memory storage. Science, 250: 288-290, 1990. 